\title{
Effects of sepsis on the metabolism of sphingomyelin and cholesterol in mice with liver dysfunction
}

\author{
JIAQI LI $^{1 *}, \mathrm{KUN} \mathrm{XIA}^{1 *}$, MINGDI XIONG $^{2^{*}}, \mathrm{XI} \mathrm{WANG}^{1}$ and NIANLONG YAN ${ }^{1}$ \\ ${ }^{1}$ Department of Biochemistry and Molecular Biology, School of Basic Medical Science; \\ ${ }^{2}$ Basic Medical Experiments Center, Nanchang University, Nanchang, Jiangxi 330006, P.R. China
}

Received February 16, 2017; Accepted July 27, 2017

DOI: $10.3892 / e t m .2017 .5226$

\begin{abstract}
Sepsis is characterized by a severe inflammatory response to infection. With the spread of sepsis, various tissues, including the lungs, liver and kidney, may be damaged. This may finally develop into multiple organ dysfunction syndrome. Sphingomyelin and cholesterol are two main lipids involved in sepsis. The metabolism of sphingomyelin and cholesterol in the livers of mice with sepsis needs to be clarified. To achieve this, the present study intraperitoneally injected mice with PBS, lipopolysaccharide (LPS; $10 \mathrm{mg} / \mathrm{kg}$ ) and LPS + pyrrolidine dithiocarbamate (PDTC; $30 \mathrm{mg} / \mathrm{kg}$ ). Subsequently, sphingomyelin and cholesterol content were measured using kits, the sphingomyelin synthase (SMS) activity was measured using thin layer chromatography, and the expression levels of SMS1 and 2, hydroxy-3-methylglutaryl-coenzyme A reductase (HMGCR), ATP binding cassette subfamily A member 1 (ABCA1), scavenger receptor class B member 1 (SR-B1) and apolipoprotein A1 (Apo A1) were determined by western blotting in the livers of mice. Results demonstrated that, in the LPS group, sphingomyelin and cholesterol content was significantly increased $(\mathrm{P}<0.001 ; \mathrm{n}=6)$, the SMS activity significantly enhanced $(\mathrm{P}<0.001 ; \mathrm{n}=6)$, the expression levels of SMS2, HMGCR, ABCA1 and SR-B1 were augmented $(\mathrm{P}<0.05 ; \mathrm{n}=6)$, and the expression of Apo A1 was decreased $(P<0.05 ; n=6)$, whereas SMS1 level only slightly increased with no statistical significance $(P>0.05 ; n=6)$, compared to the levels in the control group. However, PDTC was able to attenuate these alterations. These results indicated that sphingomyelin and cholesterol content may increase in the liver dysfunction of sepsis by increasing the expression of SMS2, HMGCR, SR-B1 and ABCA1, and downregulating Apo A1.
\end{abstract}

Correspondence to: Professor Nianlong Yan, Department of Biochemistry and Molecular Biology, School of Basic Medical Science, Nanchang University, 461 Bayi Road, Nanchang, Jiangxi 330006, P.R. China

E-mail: yannianlong@163.com

${ }^{*}$ Contributed equally

Key words: sepsis, lipopolysaccharide, sphingomyelin, cholesterol

\section{Introduction}

Sepsis is a life-threatening clinical disease induced by infection, and is characterized by systemic inflammation (1). With the development of sepsis, various organs, including the lungs, liver and kidney, may become damaged. This may finally develop into multiple organ dysfunction syndrome (2). According to the statistics of US Centers for Disease Control, there are 750,000 serious infection and subsequent disease cases in the United States each year (3). In China, the morbidity and mortality is consistent with those reported from abroad (4). Although great attention has been paid to basic research and clinical studies of sepsis in China and abroad, its incidence and mortality have remained high.

Several lipids are involved in sepsis and organ injury. Sphingosine-1-phosphate is a sphingolipid that has been demonstrated to significantly decrease inflammation in a murine model of acute lung injury induced by lipopolysaccharide (LPS) (5). The upstream pathways involved in sphingolipid synthesis include sphingomyelin (SM) (6). The biosynthesis of SM requires a series of enzymes, and sphingomyelin synthase (SMS) is the last critical enzyme. This enzyme has two isoforms (SMS1 and SMS2); SMS1 is found on the Golgi apparatus, and SMS2 exists in Golgi apparatus and plasma membranes (7). Research has indicated that SM participated in organ injury in sepsis (8-10). For example, studies by Hu et al (8) and Gowda et al (9) demonstrated that the expression and activity of SMS2 were enhanced in the lungs of mice during acute lung injury. Furthermore, SMS2-knockout mice demonstrated lower sensitivity to LPS, and attenuated nuclear factor (NF)- $\kappa \mathrm{B}$ activation and lung injury by suppressing mitogen-activated protein kinase-c-Jun $\mathrm{N}$-terminal kinase activation, compared to the wild-type mice (9). However, when D609, an inhibitor of SMS, inhibited the SMS activity or small interfering RNA knocked down the expression of SMS2, these treatments attenuated LPS-induced pulmonary artery endothelial cell (HPAEC) injury (10).

Cholesterol is another lipid involved in sepsis. In early 1993, a study by Memon et al (11) indicated that when C57BL/6J mice were injected with LPS, after $16 \mathrm{~h}$, the serum cholesterol levels were significantly increased by $\sim 41 \%$. Additionally, clinical cases have also demonstrated that cholesterol is involved in sepsis. For example, In one study, patients with sepsis with acute bacterial infection were enrolled and 
divided into two groups; one group had been treated with statins [a type of drug that inhibits the key enzyme activity of hydroxy-3-methylglutaryl-coenzyme A reductase (HMGCR) required for cholesterol biosynthesis] prior to their admission, and the other group had not been treated. Severe sepsis developed in $19 \%$ of patients in the non-statin group and in only $2.4 \%$ of the statin-treated group $(12,13)$. Additionally, prior exposure to statins may have a protective effect on the development of sepsis and decrease mortality in critically ill surgical patients (14). It is evident that cholesterol is involved in sepsis and may be a pro-inflammatory molecule in its development $(15,16)$.

The liver is an important immune and metabolic organ that is closely linked to several major biological functions, including synthesis of glycogen, proteins and lipids, inflammatory response, detoxification and blood clotting (17). Liver dysfunction has been known to occur frequently in the process of sepsis (18). Although research has indicated that SM and cholesterol are involved in sepsis, the effects of SM and cholesterol on liver dysfunction remain to be elucidated. To clarify the metabolism of SM and cholesterol in the liver during sepsis in the present study, BALB/c mice were treated with LPS (to induce sepsis), LPS + pyrrolidine dithiocarbamate (PDTC) or PBS. PDTC inhibits the activation of NF- $\kappa \mathrm{B}$ specifically by suppressing the release of the inhibitory subunit I $\mathrm{B}$ combining with $\mathrm{NF}-\kappa \mathrm{B}(19,20)$. SM and cholesterol content, SMS activity and related protein levels were measured.

\section{Materials and methods}

Animal model of sepsis. A total of 18 male BALB/c mice, weighing 26 \pm 3 g (5-6 weeks old), were obtained from the Experimental Animal Center of Nanchang University (Nanchang, China). All mice were kept under a 12-h light/dark cycle with free access to standard fodder and water. The temperature and humidity were $21 \pm 1^{\circ} \mathrm{C}$ and $65 \%$, respectively. Sepsis was induced in the mice as previously described (8). Briefly, the mice were divided into the following three groups ( $n=6 /$ group): Control, LPS and L + P (LPS + PDTC). The $\mathrm{L}+\mathrm{P}$ group were intraperitoneally injected with $30 \mathrm{mg} / \mathrm{kg}$ PDTC (Beyotime Institute of Biotechnology, Haimen, China) diluted in $50 \mu \mathrm{l}$ PBS. The control and LPS groups were intraperitoneally injected with the same PBS volume $(50 \mu \mathrm{l})$. After $1 \mathrm{~h}$, the LPS and L + P groups were intraperitoneally injected with $10 \mathrm{mg} / \mathrm{kg}$ LPS (Sigma-Aldrich; Merck KGaA, Darmstadt, Germany) dissolved in $50 \mu \mathrm{l}$ PBS. The mice in the control group only received the intraperitoneal injection of $50 \mu \mathrm{l}$ PBS.

Murine survival was monitored every hour for $24 \mathrm{~h}$. Subsequently, mice were euthanized by cervical dislocation and the serum and liver were collected for analysis. To identify the successful establishment of the sepsis model and liver dysfunction, plasma levels of interleukin (IL)-1 $\beta$ (E-EL-M0037c) and tumor necrosis factor (TNF)- $\alpha$ (E-EL-M0049c) (both from Elabscience Biotechnology Co., Ltd., Wuhan, China) were analyzed by ELISA using commercial kits, and the levels of alanine transaminase (ALT, C009-2) and aspartate transaminase (AST, C010-2) (both from Nanjing Jiancheng Bioengineering Institute, Nanjing, China) were detected using commercial kits.
The present study obtained ethical approval from the Committee on Animal Experimentation of Nanchang University (Nanchang, China), and the procedures complied with the NIH Guide for the Care and Use of Laboratory Animals (21).

Cholesterol and SM measurement. The livers of the mice were homogenized with PBS and then centrifuged at 9,659 $\mathrm{x} g$ at $4^{\circ} \mathrm{C}$ for $10 \mathrm{~min}$. The hepatic supernatant was collected and used to determine the protein concentration using a bicinchoninic acid (BCA) assay (CW0014S; Century Biotechnology Co., Ltd., Beijing, China). An equal volume mixture of chloroform/methanol $(2: 1, \mathrm{vol} / \mathrm{vol})$ was added to the supernatant to extract the total lipids. The mixture was centrifuged at $4,293 \mathrm{x} \mathrm{g}$, at $4^{\circ} \mathrm{C}$ for $10 \mathrm{~min}$. The supernatant was collected and then dried by nitrogen gas. The cholesterol content was calculated using a cholesterol assay kit (E1015; Applygen Technologies, Inc., Beijing, China), and the SM content was measured as previously described (22).

SMS activity assay. SMS activity of mouse liver was analyzed as previously described (23). Briefly, livers were homogenized in a buffer containing $50 \mathrm{mM}$ Tris- $\mathrm{HCl}, 1 \mathrm{mM}$ EDTA, 5\% sucrose and protease inhibitors. The homogenate was centrifuged at $9,659 \times \mathrm{g}$ at $4^{\circ} \mathrm{C}$ for $10 \mathrm{~min}$, and the supernatant was used to analyze SMS activity. The reaction system contained $50 \mathrm{mM}$ Tris- $\mathrm{HCl}$ (pH 7.4), 25 mM KCl, C6-NBD-ceramide $(0.1 \mathrm{mg} / \mathrm{ml}$; Invitrogen; Thermo Fisher Scientific, Inc., Waltham, MA, USA) and phosphatidylcholine $(0.01 \mathrm{mg} / \mathrm{ml})$. The mixture was incubated at $37^{\circ} \mathrm{C}$ for $2 \mathrm{~h}$. Subsequently, lipids were extracted in chloroform: Methanol (2:1, vol/vol), dried under nitrogen gas, and separated using thin layer chromatography. The plate was scanned with an autoradiography system (ChemiScope 6000 Pro; CLINX, Shanghai, China), and the intensity of each band was measured using Image-Pro Plus version 6.0 software (Media Cybernetics, Inc., Rockville, MD, USA).

Western blot analysis. Proteins from liver tissues of mice were extracted using radioimmunoprecipitation buffer (CW2333S; Century Biotechnology Co., Ltd.), and the protein concentration was measured using a BCA assay. Equal amounts of clear lysates ( $\sim 50 \mu \mathrm{g}$ protein) were separated by SDS-PAGE $(10 \%)$ and then transferred onto polyvinylidene fluoride membranes (EMD Millipore, Billerica, MA, USA). Equal transfer was validated by staining with Ponceau red. The membranes were blocked with $10 \%$ skimmed milk in Tris-buffered saline (TBS) at room temperature for $1 \mathrm{~h}$ and then incubated with primary antibodies in TBS containing $0.05 \%$ Tween-20, $2 \%$ bovine serum albumin (A8010; Solarbio Bioscience \& Technology Co., Ltd., Beijing, China) and $0.05 \%$ sodium azide overnight at $4^{\circ} \mathrm{C}$. The following antibodies were used at the indicated dilutions: SMS1 at 1:800 (A521; ABclonal Biotech Co., Ltd., Wuhan, China), SMS2 at 1:1,000 (AP9801b; Abgent Biotech Co., Ltd., Suzhou, China), apolipoprotein A1 (Apo A1; 14427-1-AP; Proteintech Group, Wuhan, China) at 1:500, ABCA1 (ATP binding cassette subfamily A member 1) at 1:300 (PB0490; Boster Biological Technology, Ltd., Wuhan, China), scavenger receptor class B member 1 (SR-B1) at 1:1,000 (21277-1-AP; Proteintech Group), HMGCR at 
A

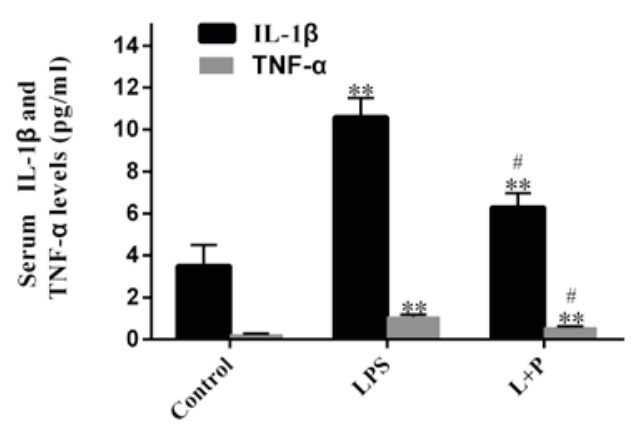

B

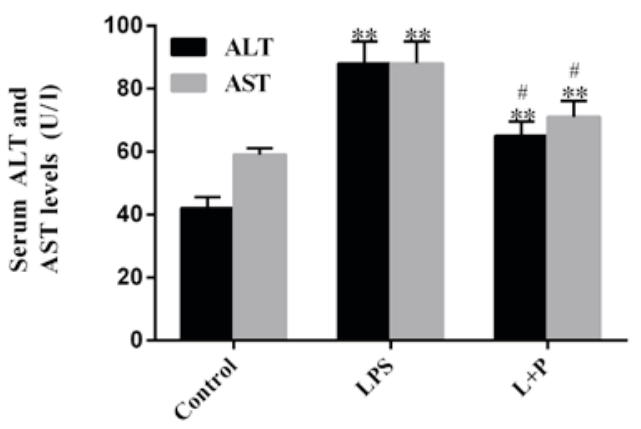

Figure 1. IL-1 $\beta$, TNF- $\alpha$, ALT and AST levels in the serum of mice. (A) Plasma levels of IL-1 $\beta$ and TNF- $\alpha$ were analyzed using ELISA kits. (B) Serum ALT and AST levels were measured. Data are presented as the mean \pm standard deviation $(n=6) .{ }^{* *} \mathrm{P}<0.001$ vs. control group; ${ }^{\#} \mathrm{P}<0.05$ vs. LPS group. IL, interleukin; TNF, tumor necrosis factor; ALT, alanine transaminase; AST, aspartate transaminase; L + P, LPS + pyrrolidine dithiocarbamate; LPS, lipopolysaccharide.

1:1,000 (A1633; ABclonal, Biotech Co., Ltd.) and $\beta$-actin at 1:10,000 (60008-1-Ig; Proteintech Group). Secondary horseradish peroxidase-coupled antibodies [mouse (SA00001-1) and rabbit (SA00001-2) (both from Proteintech Group) were used at 1:10,000 in 10\% skimmed milk in TBS containing $0.05 \%$ Tween-20 $(8,23)$. Signals were revealed using an enhanced chemiluminescence reagent (CW0049M; Century Biotechnology Co., Ltd.) and an autoradiography system (ChemiScope 6000 Pro).

Statistical analysis. Data were presented as the mean \pm standard deviation. All statistical analysis was conducted using SPSS version 17.0 software (SPSS, Inc., Chicago, IL, USA). Statistical analysis was performed using one-way analysis of variance and Tukey's post hoc test. $\mathrm{P}<0.05$ was considered to indicate a statistically significant difference.

\section{Results}

Identification of an animal model of sepsis. In the process of sepsis, the levels of plasma inflammatory factors may be increased. To identify whether the construction of an animal model of sepsis was successful, the present study measured plasma levels of IL-1 $\beta$ and TNF- $\alpha$. Results demonstrated that LPS significantly increased $(\mathrm{P}<0.001 ; \mathrm{n}=6)$ plasma levels of IL- $1 \beta$ and TNF- $\alpha$ after $24 \mathrm{~h}$, by 2.03 - and 3.69 -fold, respectively, compared to the levels in the control groups (PBS-treated; Fig. 1A). When the mice were injected with $30 \mathrm{mg} / \mathrm{kg}$ PDTC prior to treatment with LPS (L + P group), plasma levels of IL-1 $\beta$ and TNF- $\alpha$ were increased compared to the levels in the control groups (Fig. 1A; $\mathrm{P}<0.001 ; \mathrm{n}=6$ ); however, the increase folds were significantly reduced $(\mathrm{P}<0.05$; $\mathrm{n}=6 ; 0.80$ and 1.46 folds, respectively) compared with the increases induced by LPS alone. These results demonstrated that the LPS treatment was successful at inducing sepsis after $24 \mathrm{~h}$; however, PDTC was able to attenuate this process.

Furthermore, to detect whether sepsis causes damage to the liver, the AST and ALT levels were measured. Results revealed that the plasma ALT and AST levels of the LPS-treated group significantly rose by $\sim 109.59$ and $48.19 \%(\mathrm{P}<0.001 ; \mathrm{n}=6)$, respectively, compared with the levels in the control group. However, the levels in the $\mathrm{L}+\mathrm{P}$ group increased by 54.18 and $21.04 \%$, respectively, compared with the levels in the control

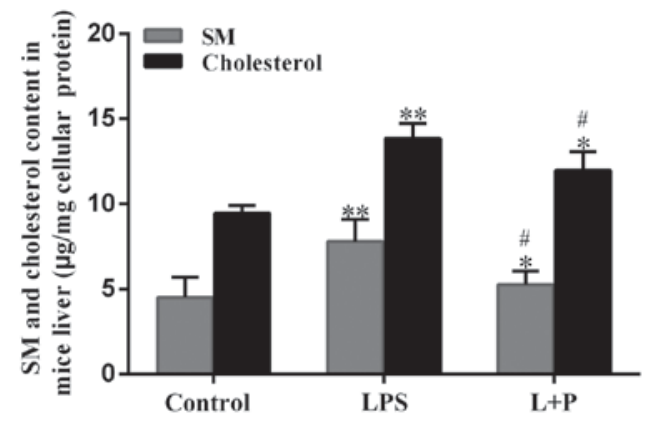

Figure 2. Content of cholesterol and SM in the livers of mice. Data are presented as the mean \pm standard deviation $(n=6)$. ${ }^{*} \mathrm{P}<0.05$ and ${ }^{* *} \mathrm{P}<0.001$ vs. control group; ${ }^{\#} \mathrm{P}<0.05$ vs. LPS group. SM, sphingomyelin; L + P, LPS + pyrrolidine dithiocarbamate; LPS, lipopolysaccharide.

group, and these levels were significantly decreased compared with the levels in the LPS group (Fig. 1B; $\mathrm{P}<0.05$; $\mathrm{n}=6$ ).

Content of cholesterol and SM in the livers of mice. SM and cholesterol are involved in the process of sepsis; therefore, the livers from the mice were homogenized with PBS to isolate the total lipids and the content of SM and cholesterol was measured. As demonstrated in Fig. 2, the SM and cholesterol content significantly increased by 73.18 and $46.74 \%$, respectively, in the LPS treatment group compared with those in the control group $(\mathrm{P}<0.001 ; \mathrm{n}=6)$. However, the $\mathrm{L}+\mathrm{P}$ treatment significantly reduced $(\mathrm{P}<0.05 ; \mathrm{n}=6)$ these levels compared with the LPS group; although the levels were still significantly greater than those in the control group $(\mathrm{P}<0.05 ; \mathrm{n}=6)$. These results suggested that the sepsis altered hepatic SM and cholesterol content, and that these peptides may be involved in sepsis-associated liver dysfunction.

SMS activity. SMS is a key enzyme of SM biosynthesis in a series of enzymatic reactions (7). Therefore, SMS activity was measured in the present study. As demonstrated in Fig. 3A, SMS activity increased significantly by $75.91 \%(\mathrm{P}<0.001$; $\mathrm{n}=6$ ) in the LPS group compared to that in the control group, and PDTC significantly reduced this increase $(\mathrm{P}<0.05 ; \mathrm{n}=6)$. As SMS1 and SMS2 may affect SMS activity, the expression levels of SMS1 and SMS2 were investigated. As indicated in Fig. 3B, the expression level of SMS2 was significantly 
A
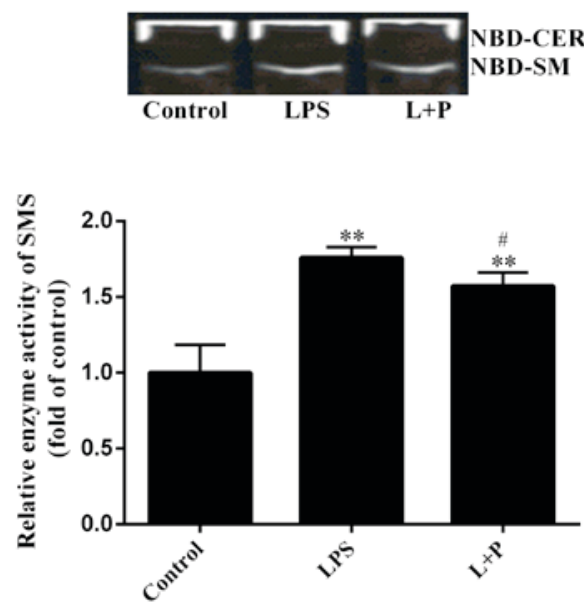

B
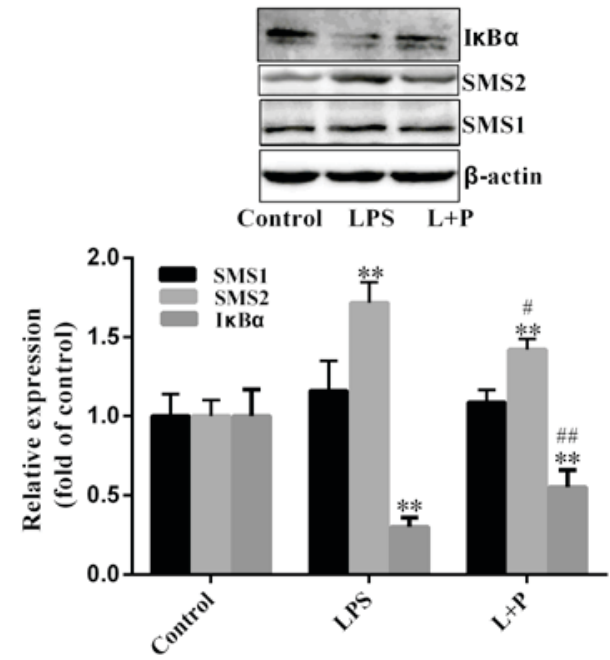

Figure 3. SMS activity and expression levels of SMS and I $\mathrm{B} \alpha$ in the livers of mice. (A) SMS activity was measured by thin layer chromatography. (B) Protein expression levels of SMS1 and 2 and $\mathrm{I} \kappa \mathrm{B} \alpha$ were analyzed by western blot analysis. Data are presented as the mean \pm standard deviation $(\mathrm{n}=6)$. ${ }^{* *} \mathrm{P}<0.001$ vs. control group; ${ }^{\#} \mathrm{P}<0.05$ and ${ }^{\# \#} \mathrm{P}<0.001$ vs. LPS group. SM, sphingomyelin; SMS, sphingomyelin synthase; CER, ceramide; L + P, LPS + pyrrolidine dithiocarbamate; LPS, lipopolysaccharide.

upregulated $(\mathrm{P}<0.001 ; \mathrm{n}=6)$ by $\sim 71.62 \%$ in the LPS treatment group compared with that in the control group. Although the expression level of SMS1 had slightly increased in the LPS group compared with the level in the control group, this difference was not statistically significant $(\mathrm{P}>0.05 ; \mathrm{n}=6)$. Notably, the increase of SMS activity (75.91\%) was similar to the increase of SMS2 expression (71.62\%). Therefore, the increase of SM content may be predominantly influenced by SMS2.

Furthermore, the protein expression level of $\mathrm{I} \kappa \mathrm{B} \alpha$ was also measured in the present study. Results indicated that the degradation level of $\mathrm{I} \kappa \mathrm{B} \alpha$ was significantly increased in the LPS-treated group (Fig. 3B; $\mathrm{P}<0.001 ; \mathrm{n}=6$ ) compared to that in the control group; however, PDTC significantly inhibited $(\mathrm{P}<0.001 ; \mathrm{n}=6)$ the $\mathrm{I} \kappa \mathrm{B} \alpha$ degradation compared to the LPS group. These results suggested that PDTC inhibition of NF- $\kappa \mathrm{B}$ activity may reduce hepatic inflammation.

Expression of cholesterol-related proteins. In cells, HMGCR is a key enzyme in the biosynthesis of cholesterol, however, the transportation of cholesterol is associated with Apo A1, ABCA1 and SR-B1 (24). Therefore, the expression levels of these proteins in the livers of mice were analyzed. As demonstrated in Fig. 4, HMGCR was significantly upregulated $(\mathrm{P}<0.05 ; \mathrm{n}=6)$ by $\sim 37.63 \%$ in the LPS group compared with the level in the control group. Furthermore, ABCA1 and SR-B1 expression levels were significantly upregulated $(\mathrm{P}<0.001$; $\mathrm{n}=6$ ) in the LPS group, with an increase of $\sim 57.82$ and $111.70 \%$, respectively, compared to the levels in the control group. The expression level of Apo A1 was significantly downregulated $(\mathrm{P}<0.001 ; \mathrm{n}=6)$ by $\sim 43.31 \%$ in the LPS group compared with the level in the control group. However, when PDTC was administered prior to LPS treatment, the trend of upregulation or downregulation was significantly reversed $(\mathrm{P}<0.05 ; \mathrm{n}=6)$ compared with the LPS group. These results suggested that depression of $\mathrm{NF}-\kappa \mathrm{B}$ by PDTC may reduce the alterations induced by LPS.

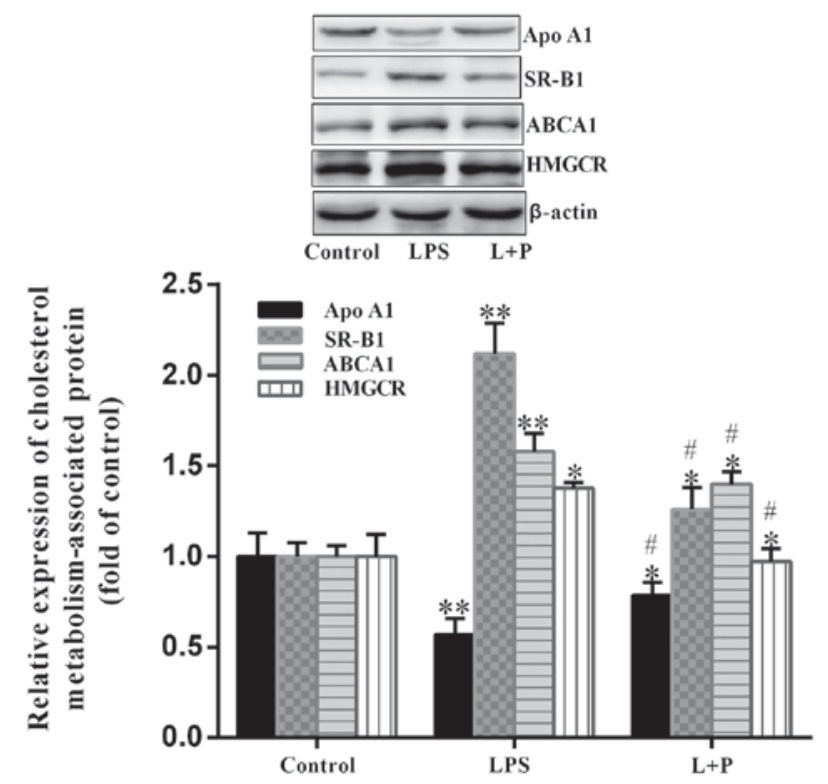

Figure 4. Expression of HMGCR, ABCA1, SR-B1 and Apo A1 in the livers of mice. Protein expression levels of ABCA1, HMGCR, SR-B1 and Apo A1 were analyzed by western blot analysis. Data are presented as the mean \pm standard deviation $(\mathrm{n}=6)$. ${ }^{*} \mathrm{P}<0.05$ and ${ }^{* *} \mathrm{P}<0.001$ vs. control group; ${ }^{*} \mathrm{P}<0.05$ vs. LPS group. L + P, LPS + pyrrolidine dithiocarbamate; LPS, lipopolysaccharide; HMGCR, hydroxy-3-methylglutaryl-coenzyme A reductase; ABCA1, ATP binding cassette subfamily A member 1; SR-B1, scavenger receptor class B member 1; Apo A1, apolipoprotein A1.

\section{Discussion}

LPS-induced sepsis is a complex process that may damage many organs, particularly the lungs, liver and kidney, and several lipids are involved in this process (1). However, in murine livers, the effect of sepsis on regulation of SM and cholesterol metabolism remains unknown, and it is necessary to clarify it. The results of the present study demonstrated that LPS treatment was able to induce sepsis and liver dysfunction 
in mice. Following LPS treatment, SM and cholesterol content was increased, and the protein expression levels of HMGCR, ABCA1, SR-B1 and SMS2 were increased. Contrastingly, the expression of Apo A1 was downregulated compared to the control group. However, when mice were intraperitoneally injected with PDTC, which may attenuate the inflammatory response by inhibiting $\mathrm{NF}-\kappa \mathrm{B}$, all of the changes induced by LPS were reversed $(19,20)$. These results indicated that sepsis directly affected the SM and cholesterol content, and the expression of these proteins in the livers of mice.

Various reports have demonstrated that SMS was involved in the inflammatory process. Studies by Hailemariam et al (25) and Gowda et al (9) indicated that LPS treatment significantly increased SMS2 enzyme activity in HPAEC cells. Furthermore, adding the SMS inhibitor (D609) or knocking out SMS2 may attenuate inflammation in macrophages and lung injury of mice induced by LPS $(9,10)$. In other words, SMS2 participates in and promotes sepsis. In the present study, it was indicated that hepatic SMS activity increased following LPS treatment, and this implied that the cause of the increase in SMS activity and SM content was the overexpression of SMS2, but not of SMS1. SMS2 is the principal enzyme that takes charge of the biosynthesis of SM in the liver (26).

Cholesterol is a main lipid in cells that participates in sepsis. For example, modified low-density lipoprotein (oxLDL) treatment of macrophages increases cholesterol content and triggers inflammation in vivo, and mice fed with a high-fat diet also demonstrate inflammatory properties in macrophages (27). However, simvastatin may depress the inflammatory response by reducing cholesterol content (27). When mice are injected with LPS, the sepsis and liver dysfunction would be induced in the body of the mice, and the cholesterol content would be enhanced in the liver. The cholesterol content is tightly associated with cholesterol efflux in the livers of mice (24). ABCA1 is a transmembrane protein that is able to mediate cholesterol efflux; however, Apo A1 may accept the cholesterol by interacting with ABCA1 to form the high-density lipoprotein (HDL), with a series of changes, and finally, the HDL may become the mature HDL $(24,28)$. Therefore, the overexpression of ABCA1 and Apo A1 may strengthen the cholesterol efflux and decrease the content of cholesterol in the liver (24). However, in the present study, the cholesterol content and the expression of ABCA1 were increased following LPS treatment. In early 2002, a study by Kaplan et al (29) demonstrated the same results as the present study. They believed that LPS may rapidly increase the expression of ABCA1 in the liver and THP-1 cells through a liver X receptor-independent mechanism (29). However, we suggest that the upregulation of ABCA1 expression in liver cells may reflect a feedback inhibition mechanism by elevating cellular cholesterol content. Furthermore, reasonable explanations for the increase of the cholesterol content may be as follows: i) Elevated expression of HMGCR may increase the biosynthesis of cholesterol; ii) as an HDL receptor on liver cell membranes, SR-B1 may take up cholesterol into the liver (25), and the increasing SR-B1 expression may promote cholesterol uptake and cholesterol influx; and iii) the downregulation of Apo A1 may also attenuate the cholesterol efflux, and accumulate the cholesterol in the livers of mice.
SM and cholesterol are main components of lipid rafts, which are sites for numerous cellular processes, including signaling, vesicular transporting, interaction with pathogens and viral infection (30). In lipid rafts, SM and cholesterol may be anchored with each other, therefore, they may simultaneously increase in the livers of mice, and only SM and cholesterol were detected in the liver in the present study. For example, studies by Ding et al (31) and Yan et al (32) demonstrated that SMS overexpression may cause SM accumulation in cells and lipid rafts and increase the cholesterol content. Furthermore, patients with Niemann-Pick disease cannot hydrolyze SM due to defective SMase, resulting in the accumulation of $\mathrm{SM}$ and cholesterol in the liver and the nervous system (33). Additionally, because ABCA1 is a transmembrane protein that is located in or near the lipid raft, some researchers have implied that the enhancement of SM and cholesterol may also increase the ABCA1 expression in cells (32).

In conclusion, in the livers of mice, both SM and cholesterol content were increased during sepsis induced by LPS; however, PDTC was able to attenuate these alterations. Further investigation indicated that the change of SM and cholesterol were tightly associated with the overexpression of SMS2, HMGCR, SR-B1 and ABCA1, and the downregulation of Apo A1. These results suggested that lipid metabolism is a key factor affecting sepsis.

\section{Acknowledgements}

The present research was supported by a grant from the Jiangxi Provincial Department of Science and Technology (grant no. 20142BAB205014).

\section{References}

1. Zotova NV, Chereshnev VA and Gusev EY: Systemic inflammation: Methodological approaches to identification of the common pathological process. PLoS One 11: e0155138, 2016.

2. Dombrovskiy VY, Martin AA, Sunderram J and Paz HL: Rapid increase in hospitalization and mortality rates for severe sepsis in the United States: A trend analysis from 1993 to 2003. Crit Care Med 35: 1244-1250, 2007.

3. Tadros T, Traber DL, Heggers JP and Herndon DN: Effects of interleukin-1alpha administration on intestinal ischemia and reperfusion injury, mucosal permeability, and bacterial translocation in burn and sepsis. Ann Surg 237: 101-109, 2003.

4. Chen XC, Yang YF, Wang R, Gou HF and Chen XZ: Epidemiology and microbiology of sepsis in mainland China in the first decade of the 21st century. Int J Infect Dis 31: 9-14, 2015.

5. Peng X, Hassoun PM, Sammani S, McVerry BJ, Burne MJ, Rabb H, Pearse D, Tuder RM and Garcia JG: Protective effects of sphingosine 1-phosphate in murine endotoxin-induced inflammatory lung injury. Am J Respir Crit Care Med 169: 1245-1251, 2004.

6. Bektas M, Allende ML, Lee BG, Chen W, Amar MJ, Remaley AT, Saba JD and Proia RL: Sphingosine-1-phosphatelyase deficiency disrupts lipid homeostasis in liver. J Biol Chem 285: 10880-10889, 2010.

7. Yeang C, Ding T, Chirico WJ and Jiang XC: Subcellular targeting domains of sphingomyelin synthase 1 and 2 . Nutr Metab (Lond) 8: 89, 2011.

8. Hu S, Ding Y, Gong J and Yan N: Sphingomyelin synthase 2 affects CD14-associated induction of NF- $\mathrm{kB}$ by lipopolysaccharides in acute lung injury in mice. Mol Med Rep 14: 3301-3306, 2016.

9. Gowda S, Yeang C, Wadgaonkar S, Anjum F, Grinkina N, Cutaia M, Jiang XC and Wadgaonkar R: Sphingomyelin synthase 2 (SMS2) deficiency attenuates LPS-induced lung injury. Am J Physiol Lung Cell Mol Physiol 300: L430-L440, 2011 . 
10. Anjum F, Joshi K, Grinkina N, Gowda S, Cutaia M and Wadgaonkar R: Role of sphingomyelin synthesis in pulmonary endothelial cell cytoskeletal activation and endotoxin-induced lunginjury. Am J Respir Cell Mol Biol 47: 94-103, 2012.

11. Memon RA, Grunfeld C, Moser AH and Feingold KR: Tumor necrosis factor mediates the effects of endotoxin on cholesterol and triglyceride metabolism in mice. Endocrinology 132: 2246-53, 1993.

12. Ozguler IM, Burma O, Uysal A and Akbulut H: Rosuvastatin lowers systemic inflammatory response in coronary artery bypass graft accompanied by cardiopulmonary bypass surgery: A randomized controlled study. Clin Invest Med 38: E154-E163, 2015.

13. Almog Y, Shefer A, Novack V, Maimon N, Barski L, Eizinger M, Friger M, Zeller L and Danon A: Prior statin therapy is associated with a decreased rate of severe sepsis. Circulation 110: 880-885, 2004

14. Schurr JW, Wu W, Smith-Hannah A, Smith CJ and Barrera R Incidence of sepsis and mortality with prior exposure of HMG-COA reductase inhibitors in a surgical intensive care population. Shock 45: 10-15, 2016.

15. Chung S, Cuffe H, Marshall SM, McDaniel AL, Ha JH, Kavanagh K, Hong C, Tontonoz P, Temel RE and Parks JS: Dietary cholesterol promotes adipocyte hypertrophy and adipose tissue inflammation in visceral, but not in subcutaneous, fat in monkeys. Arterioscler Thromb Vasc Biol 34: 1880-1887, 2014

16. Lewis GF and Rader DJ: New insights into the regulation of HDL metabolism and reverse cholesterol transport. Circ Res 96: $1221-1232,2005$

17. Guo K, Ren J, Wang G, Gu G, Li G, Wu X, Chen J, Ren H, Hong Z, Wu L, et al: Early liver dysfunction in patients with intra-abdominal infections. Medicine (Baltimore) 94: e1782, 2015.

18. Ding R, Han J, Zhao D, Hu Z and Ma X: Pretreatment with Rho-kinase inhibitor ameliorates lethal endotoxemia-induced liver injury by improving mitochondrial function. Int Immunopharmacol 40: 125-130, 2016.

19. Kabay S, Ozden H, Guven G, Burukoglu D, Ustuner MC, Topal F, Gunes HV, Ustuner D and Ozbayer C: Protective effects of the nuclear factor kappa B inhibitor pyrrolidine dithiocarbamate on experimental testicular torsion and detorsion injury. Korean $\mathrm{J}$ Physiol Pharmaco 1 18: 321-326, 2014.

20. Cuzzocrea S, Chatterjee PK, Mazzon E, Dugo L, Serraino I, Britti D, Mazzullo G, Caputi AP and Thiemermann C: Pyrrolidine dithiocarbamate attenuates the development of acute and chronic inflammation. Br J Pharmacol 135: 496-510, 2002.

21. National Research Council (US) Committee for the Update of the Guide for the Care and Use of Laboratory Animals: Guide for the Care and Use of Laboratory Animals. National Academies Press (US), Washington, DC, 2011.
22. Dong J, Liu J, Lou B, Li Z, Ye X, Wu M and Jiang XC Adenovirus-mediated overexpression of sphingomyelin synthase 1 and 2 increases the atherogenic potential in mice. J Lipid Res 47: 1307-1014, 2006.

23. Liu AQ, Xie Z, Chen XN, Feng J, Chen JW, Qin FJ and Ge LY Fas-associated factor 1 inhibits tumor growth by suppressing Helicobacter pylori-induced activation of NF- $\kappa \mathrm{B}$ signaling in human gastric carcinoma. Oncotarget 8: 7999-8009, 2017.

24. Lewis GF and Rader DJ: New insights into the regulation of HDL metabolism and reverse cholesterol transport. Circ Res 96 1221-1232, 2005.

25. Hailemariam TK, Huan C, Liu J, Li Z, Roman C, Kalbfeisch M, Bui HH, Peake DA, Kuo MS, Cao G, et al: Sphingomyelin synthase 2 deficiency attenuates NFkappaB activation. Arterioscler Thromb Vasc Biol 28: 1519-1526, 2008.

26. Liu J, Zhang H, Li Z, Hailemariam TK, Chakraborty M, Jiang K, Qiu D, Bui HH, Peake DA, Kuo MS, et al: Sphingomyelin synthase 2 Is one of the determinants for plasma and liver sphingomyelin levels in mice. Arterioscler Thromb Vasc Biol 29: 850-856, 2009.

27. Ho PC, Chang KC, Chuang YS and Wei LN: Cholesterol regulation of receptor interacting protein 140 via microRNA-33 in inflammatory cytokine production. FASEB J 25: 1758-1766, 2011.

28. Segrest JP, Jones MK, De Loof H, Brouillette CG, Venkatachalapathi YV and Anantharamaiah GM: The amphipathic helix in the exchangeable apolipoproteins: A review of secondary structure and function. J Lipid Res 33: 141-166, 1992.

29. Kaplan R, Gan X, Menke JG, Wright SD and Cai TQ: Bacterial lipopolysaccharide induces expression of ABCA1 but not ABCG1 via an LXR-independent pathway. J Lipid Res 43: 952-959, 2002.

30. Simons K and Ikonen E: Functional rafts in cell membranes. Nature 387: 569-572, 1997.

31. Ding TB, Li ZQ, Hailemariam T, Mukherjee S, Maxfield FR, Wu MP and Jiang XC: SMS overe-xpression and knockdown: Impact on cellular sphingomyelin and diacylglycerol metabolism and cell apoptosis. J Lipid Res 49: 376-385, 2008.

32. Yan N, Ding T, Dong J, Li Y and Wu M: Sphingomyelin synthase overexpression increases cholesterol accumulation and decreases cholesterol secretion in liver cells. Lipids Health Dis 10: 46, 2011.

33. Lee CY, Lesimple A, Denis M, Vincent J, Larsen A, Mamer O, Krimbou L, Genest J and Marcil M: Increased sphingomyelin content impairs HDL biogenesis and maturation in human Niemann-Pick disease type B. J Lipid Res 47: 622-632, 2006. 